\title{
Hüftgelenkersatz beim Rheumatiker
}

D ie durch das rheumatische Grundleiden zerstörten Hüftgelenke bedeuten für den Patienten in der Regel eine erheblich schwerere Invalidität gegenüber derjenigen, wie wir sie beispielsweise bei $\mathrm{Pa}$ tienten mit primärer Koxarthrose feststellen. Patienten, die an einer destruierenden rheumatischen Koxitis leiden, sind nicht nur von belastungsabhängigen Schmerzen geplagt, sondern meist auch von Ruhe- und Nachtschmerzen. Für den orthopädischen Chirurgen stellt das Einsetzen eines künstlichen Hüftgelenks beim Rheumatiker nicht selten eine größere Herausforderung dar, weil die Fixation von Prothesenschaft und pfanne wegen der beim Rheumatiker häufig feststellbaren schlechteren Knochenqualität, aber auch wegen der oft ausgedehnten Destruktion der knöchernen Pfanne schwieriger ist und nicht selten zusätzliche Maßnahmen erfordert. Hinzu kommt wegen des nicht selten polyartikulären Befalls die beim Rheumatiker schwierigere und langdauernde postoperative Rehabilitation. Um die Ergebnisse der beim Rheumapatienten eingesetzten Hüfttotalendoprothesen (Hüft-TEP) an einer größeren Fallzahl zu untersuchen, wurde von der ARO (ehemaliger DGOT-Arbeitskreis Rheumaorthopädie) eine Multicenterstudie durchgeführt, an der sich $24 \mathrm{Kli}$ niken beteiligten. In der 1995/1996 abgeschlossenen Studie sollten alle 1987/1988 mit einer Hüft-TEP versorgten Rheumapatienten erfaßt werden. Zum Vergleich wurden die in der gleichen Klinik im gleichen Zeitraum wegen einer primä- ren Koxarthrose oder einer Dysplasiekoxarthrose operierten Patienten in die Untersuchung einbezogen; die Dysplasiekoxarthrose deswegen, weil bei ihr die anatomischen Voraussetzungen an Schaft und vor allem der Pfanne bekanntermaßen schwieriger sind als bei der primären Koxarthrose.

Die Ergebnisse der Studie sind in den ersten 3 Übersichtsartikeln dieses Hefts wiedergegeben, wobei Arnold et al. über die vergleichenden klinischen Ergebnisse und Schroeder-Boersch et al. über die radiologischen Ergebnisse berichten. Schüle et al. gehen im einzelnen noch einmal auf die Versageranalyse der in der Studie gewählten Implantate ein. Das erstaunliche und zugleich erfreuliche Fazit der Studie war, daß die Rheumapatienten in keiner Weise eindeutig schlechtere Resultate als die beiden anderen Diagnosegruppen aufwiesen und erstaunlicherweise auch nicht bei der zementfreien Implantation, sofern in den Kliniken nicht Implantate verwendet wurden, die sich bereits Ende der 8oer und Anfang der goer Jahre als ungeeignet erwiesen haben und inzwischen vom Markt verschwunden sind.

$\mathrm{Da}$ die ARO-Studie gezeigt hat, daß bei den Versagern insbesondere die Pfannenimplantation herausragte, wurden in dieses Heft 4 weitere Artikel aufgenommen, die sich mit der Pfannenproblematik bei rheumatoider Arthritis eingehend befassen. So haben Dominkus et al. ihre Methode der Pfannenmigrationsanalyse wiedergegeben, mit der frühzeitige Pfannenlockerungen besser erkennbar werden. Effenberger et al. berichten über verschiedene zementfreie implantierte Pfannentypen, während Starker et al. ihre Methode der Pfannenrekonstruktion mit Pfannenstützscha- len aufzeigen. Von Sparmann wird schließlich über die erheblichen technischen Probleme bei den Drei- und Vierfachwechseloperationen der Hüftpfanne berichtet.

$D a$ die ARO-Studie zwangsläufig nur mittelfristige Resultate wiedergeben konnte, wurden von Wessinghage und Kisslinger die Langzeitergebnisse nach zementierten Prothesen und von $\mathrm{Lu}$ koschek die Ergebnisse zementfreier Hüftendoprothesen jeweils bei rheumatischer Grundkrankheit in dieses Heft integriert.

Allen Autoren und Koautoren, insbesondere aber allen Mitarbeiterinnen und Mitarbeitern in den ARO-Kliniken (die Namen sind in der nachfolgenden Arbeit von Arnold et al. aufgelistet), sei an dieser Stelle herzlichst gedankt für ihren großartigen Einsatz bei der Nachuntersuchung der Patienten und der Auswertung der Ergebnisse.
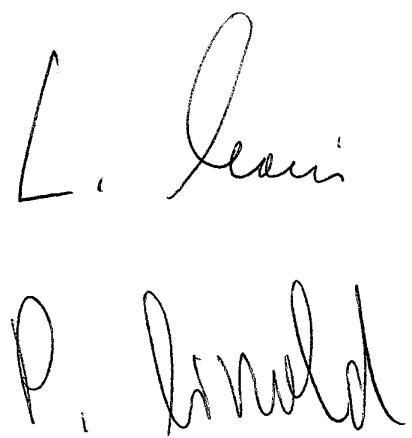

Lutz Jani/Peter Arnold Die Herausgeber 